CONCISE REPORT

\title{
Pomalidomide is effective for prevention and treatment of experimental skin fibrosis
}

\author{
Simon Weingärtner, ${ }^{1}$ Pawel Zerr, ${ }^{1}$ Michal Tomcik, ${ }^{1}$ Katrin Palumbo-Zerr, ${ }^{1}$ Alfiya Distler, ${ }^{1}$ \\ Clara Dees, ${ }^{1}$ Christian Beyer, ${ }^{1}$ Sai L Shankar, ${ }^{2}$ Dorota Cedzik, ${ }^{2}$ Peter H Schafer, ${ }^{2}$ \\ Oliver Distler, ${ }^{3}$ Georg Schett, ${ }^{1}$ Jörg H W Distler ${ }^{1}$
}

\begin{abstract}
- Additional data are published online only. To view this file please visit the journal online (http://dx.doi org/10.1136/annrheumdis2012-201784

${ }^{1}$ Department of Internal Medicine 3 and Institute for Clinical Immunology, University of Erlangen-Nuremberg, Erlangen, Germany

${ }^{2}$ Celgene Corporation, Translational Development,

Summit, New Jersey, USA

${ }^{3}$ Department of Rheumatology,

University Hospital Zurich,

Zürich, Switzerland
\end{abstract}

\section{Correspondence to}

Dr Jörg Distler, Department of

Medicine 3 and Institute for

Clinical Immunology, University

of Erlangen-Nuremberg,

Ulmenweg 18, Erlangen

D-91054, Germany;

joerg.distler@uk-erlangen.de

Accepted 17 July 2012

\section{ABSTRACT}

Objectives Tissue fibrosis is a major hallmark and a leading cause of death in systemic sclerosis (SSc). Here, we investigated the antifibrotic effects of pomalidomide, an analogue of thalidomide with potent immunomodulatory effects, in preclinical models of skin fibrosis.

Methods We evaluated the antifibrotic effects of pomalidomide in preventive as well as therapeutic treatment regimes using bleomycin-induced dermal fibrosis as a model of early, inflammatory stages of fibrosis and the tight-skin mouse model as a model of later stages of fibrosis with endogenous activation of fibroblasts.

Results Treatment with pomalidomide in doses from 0.3 to $30 \mathrm{mg} / \mathrm{kd} /$ day prevented skin fibrosis in Tsk-1 mice and in bleomycin-induced dermal fibrosis in a dose-dependent manner and reduced the expression of transforming growth factor (TGF) $\beta$-target genes such as PAI-1, CTGF and col 1a1. Pomalidomide was also effective in the setting of preestablished fibrosis and reduced dermal thickness, myofibroblast counts and hydroxyproline content below pretreatment levels.

Conclusions We demonstrate for the first time that pomalidomide exerts potent antifibrotic effects in different preclinical models of skin fibrosis. These findings lend preclinical support for the clinical studies of pomalidomide in SSc.

\section{INTRODUCTION}

Skin fibrosis is the major hallmark of systemic sclerosis (SSc). During the course of the disease, progression of fibrosis disrupts physiological tissue architecture and causes high morbidity among patients with SSc. ${ }^{1}$

IMiDs are structural and functional analogues of thalidomide that represent a new class of immunomodulators that are currently evaluated for treatment of several of autoimmune and neoplastic diseases. ${ }^{2}$ Pomalidomide (CC-4047) is the latest member of second generation IMiDs. Second generation IMiDs exhibit a greatly enhanced immunomodulatory effect in preclinical studies compared with the parental drug thalidomide. ${ }^{3}$ In addition to increased potency, the side-effect profile of pomalidomide is improved and common side-effects of thalidomide such as sedation and neurotoxicity occurred less frequently in patients treated with pomalidomide. The molecular mechanisms underlying the immunomodulatory effects of IMiDs are incompletely understood and may vary for different autoimmune diseases. Proposed mechanisms include inhibition of NFKB signalling, activation of natural killer cells and cytotoxic T cells, modulation of the expression of adhesion molecules as well as shifts from a $\mathrm{T}$ helper cell (Th)2 to a Th1 response. $^{4-7}$

We demonstrate in the present study that treatment with pomalidomide prevents fibrosis in different preclinical models of scleroderma and induces regression of pre-established experimental fibrosis. These findings had direct translational implications and stimulated the initiation of an international, multicentre, controlled phase II clinical trial to evaluate the safety and the tolerability of pomalidomide in SSc, which has most recently been launched (clinicaltrials.gov ID: NCT01559129).

\section{MATERIALS AND METHODS \\ Prevention of bleomycin-induced dermal fibrosis}

Skin fibrosis was induced in 6-week-old DBA/2 mice (Janvier SAS, Le Genest Saint Isle, France) by bleomycin as described with imatinib as positive control. $^{8} 9$ Pomalidomide (kindly provided by Celgene Corporation, Summit, New Jersey, USA) was dissolved in dimethyl sulfoxide (DMSO) at a stock concentration of $200 \mathrm{mg} / \mathrm{ml}$ and further suspended in $\mathrm{ddH} 2 \mathrm{O} / 0.5 \%$ carboxymethylcellulose/ $0.25 \%$ tween 80 to final concentrations of $0.3,3$ and $30 \mathrm{mg} / \mathrm{kg}$ oral daily, which was given by oral gavage. Sham treatment was performed with 3\% DMSO/ $0.5 \%$ carboxymethylcellulose $/ 0.25 \%$ tween 80 .

\section{Established bleomycin-induced dermal fibrosis}

A modified bleomycin-model was used to study the effectiveness of pomalidomide for the treatment of established fibrosis. ${ }^{8} 10$ The following groups were analysed: (1) $\mathrm{NaCl}$ subcutaneous for 6 weeks and sham treatment for 6 weeks; (2) bleomycin subcutaneous for 3 weeks followed by $\mathrm{NaCl}$ for 3 weeks, sham treatment for 6 weeks; (3) bleomycin subcutaneous for 6 weeks and sham treatment for 6 weeks; (4) bleomycin subcutaneous for 6 weeks and pomalidomide $0.3 \mathrm{mg} / \mathrm{kg}$ oral daily for 6 weeks; (5) bleomycin subcutaneous for 6 weeks and pomalidomide $3.0 \mathrm{mg} / \mathrm{kg}$ oral daily for 6 weeks; and (6) bleomycin subcutaneous for 6 weeks and pomalidomide $30 \mathrm{mg} / \mathrm{kg}$ oral daily for 6 weeks.

\section{Tsk-1 mice}

Tsk-1 mice develop spontaneous fibrosis of the hypodermal layers. ${ }^{8}$ Treatment of Tsk- 1 as well as 
$\mathrm{pa} / \mathrm{pa}$ control mice started at the age of 5 weeks and was continued for 5 weeks. ${ }^{11}$ The following groups were examined: (1) $\mathrm{pa} / \mathrm{pa}$ and sham treatment; (2) Tsk-1 and sham treatment; (3) Tsk-1 and pomalidomide $0.3 \mathrm{mg} / \mathrm{kg}$ oral daily; (4) Tsk-1 and pomalidomide $3.0 \mathrm{mg} / \mathrm{kg}$ oral daily; and (5) Tsk-1 and pomalidomide $30.0 \mathrm{mg} / \mathrm{kg}$ oral daily.

\section{Analysis of murine skin}

Dermal thickness, hydroxyproline content and $\alpha$-smooth muscle actin were analysed as described. ${ }^{9} 1112$

\section{Statistical analysis}

Data are expressed as the mediantinterquartile range. Wilcoxon-Mann-Whitney test was used for statistical analysis of related and non-related samples. $p$ Values less than 0.05 were considered as significant.

\section{RESULTS}

\section{Treatment of mice with pomalidomide is well tolerated}

Treatment with pomalidomide in doses up to $30 \mathrm{mg} / \mathrm{kg}$ oral daily for 3 weeks was well tolerated. Pomalidomide-treated mice appeared healthy with normal activity, behaviour and texture of the fur. The body weight did also not differ between mice treated with pomalidomide and sham-treated controls.

\section{Pomalidomide exerts antifibrotic effects in Tsk-1 mice}

Treatment with pomalidomide exerted potent antifibrotic effects in the Tsk-1 mouse model (figure 1A). Dose-dependent reductions in hypodermal thickness were observed in Tsk-1 mice treated with pomalidomide in doses between 0.3 and $30 \mathrm{mg} / \mathrm{kg}$ oral daily (figure $1 \mathrm{~B}$ ). Maximal effects with a $54 \% \pm 6 \%(p=0.001)$ were observed at doses of $30 \mathrm{mg} / \mathrm{kg}$ oral daily. Consistent with decreased hypodermal thickness, pomalidomide also prevented differentiation of resting fibroblasts into myofibroblasts (figure 1C) and reduced the hydroxyproline content in fibrotic skin (figure 1D). The antifibrotic effects of pomalidomide at doses of $30 \mathrm{mg} / \mathrm{kg}$ oral daily were comparable with those obtained with imatinib.

\section{Pomalidomide prevents bleomycin-induced dermal fibrosis}

Pomalidomide prevented bleomycin-induced dermal fibrosis in a dose-dependent manner when initiated together with the first injection of bleomycin (see online supplementary figure 1a). Although doses of $0.3 \mathrm{mg} / \mathrm{kg}$ oral daily did not prevent dermal thickening, pomalidomide in doses of 3 and $30 \mathrm{mg} / \mathrm{kg}$ oral daily significantly decreased the dermal thickness compared with sham-treated mice (see online supplementary figure $1 \mathrm{~b}$ ). Pomalidomide also prevented myofibroblast differentiation (see online supplementary figure 1c) as well as the bleomycin-induced increase in hydroxyproline content (see online supplementary figure 1d).

\section{Pomalidomide induces regression of pre-established bleomycin-induced dermal fibrosis}

We next investigated whether pomalidomide may also be effective in a therapeutic setting, when initiated after fibrosis
A
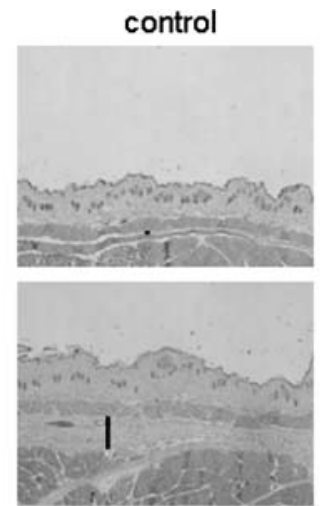

Pom $3 \mathrm{mg} / \mathrm{kg}$

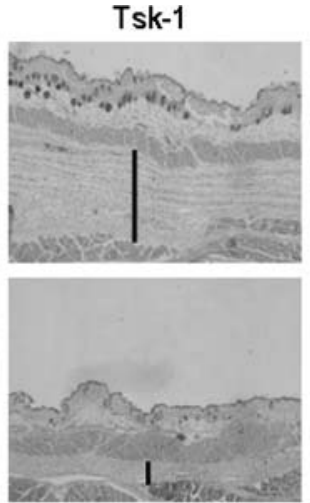

Pom $30 \mathrm{mg} / \mathrm{kg}$

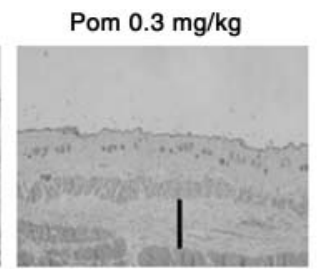

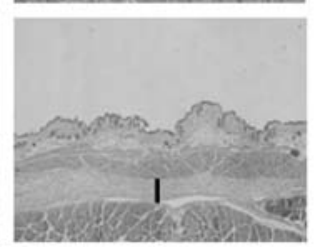

Imatinib $30 \mathrm{mg} / \mathrm{kg}$

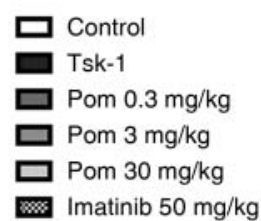

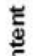

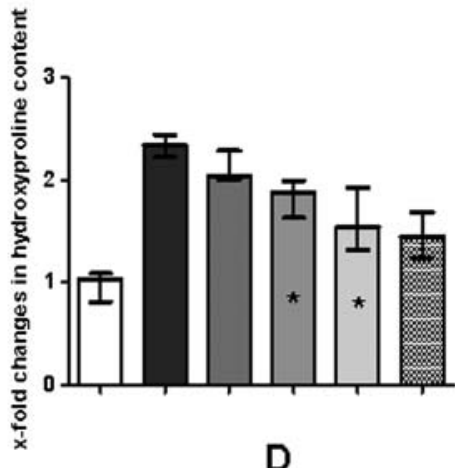

Figure 1 Pomalidomide(Pom)ameliorates fibrosis in Tsk-1 mice. Treatment with Pom at the indicated doses exerted potent antifibrotic effects in the Tsk-1 model (A) and dose-dependently reduced hypodermal thickening (B), decreased myofibroblast counts (C) and reduced the hydroxyproline content (D). Representative images of H\&E-stained skin sections are shown at 40 -fold magnification. $\mathrm{N}=8$ for all groups. Black bars indicate the hypodermal thickness. ${ }^{*}$ Indicates statistically significant changes compared with sham-treated Tsk-1 mice. 

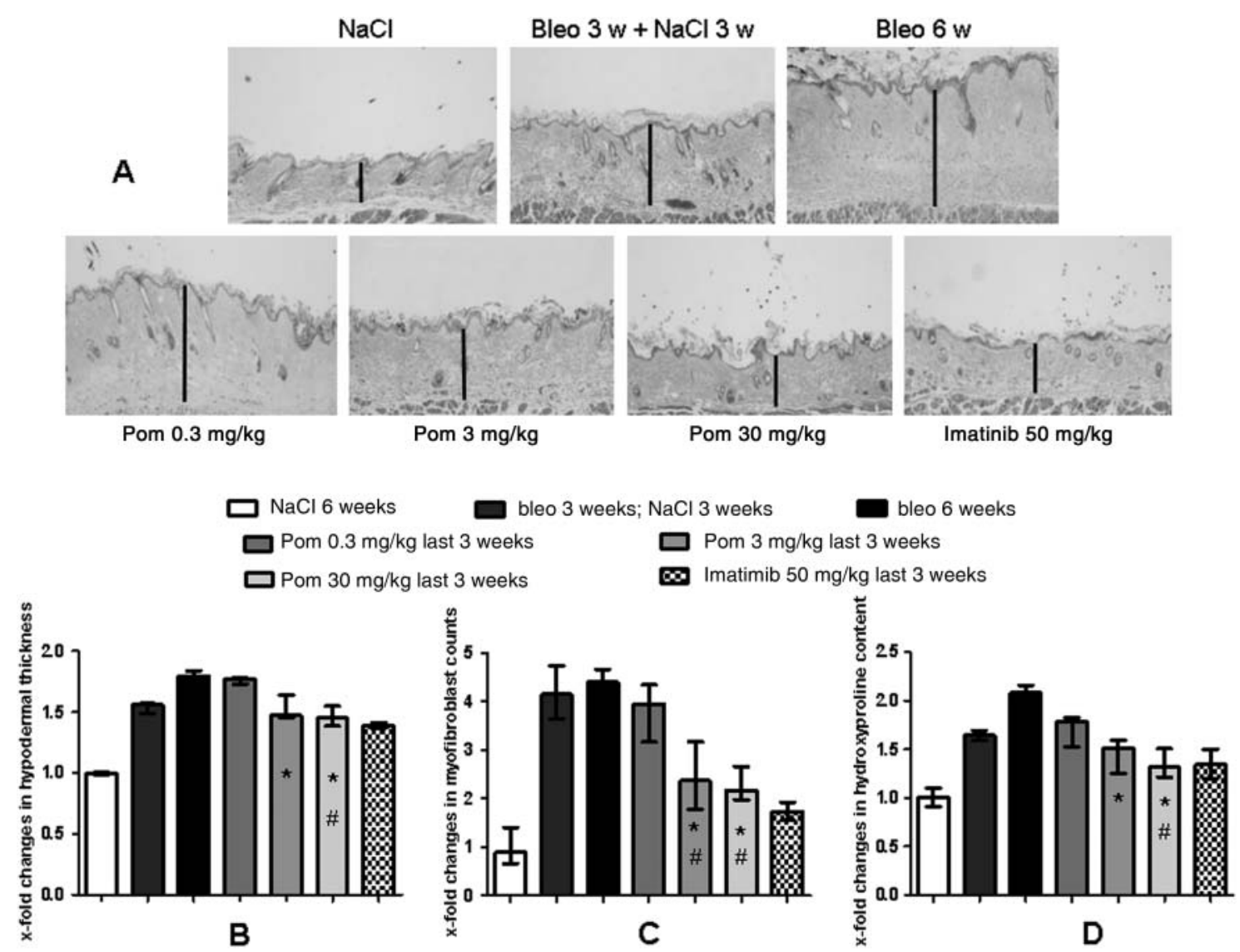

Figure 2 Pomalidomide(Pom)induces regression of bleomycin (bleo)-induced dermal fibrosis. Pom exerted dose-dependent antifibrotic effects in the setting of pre-established bleo-induced dermal fibrosis (A). Doses of $0.3 \mathrm{mg} / \mathrm{kg}$ oral daily Pom completely prevent progression of fibrosis despite ongoing challenge with bleo and doses of $30 \mathrm{mg} / \mathrm{kg}$ oral daily induced regression of bleo-induced fibrosis with decreases in dermal thickness (B), myofibroblast counts (C) and hydroxyproline levels (D) below pretreatment levels represented by sham-treated mice challenged with bleo for 3 weeks followed by injections of $\mathrm{NaCl}$ for another 3 weeks (figure $3 \mathrm{~A}$ ). Representative images of H\&E-stained skin sections are shown at 100-fold magnification. $\mathrm{N}=8$ for all groups. Black bars indicate the dermal thickness. * Indicates statistically significant changes compared with sham-treated mice challenged with bleo for 6 weeks. ${ }^{\#}$ Indicates statistically significant changes compared with sham-treated mice challenged with bleo for 3 weeks followed by injections of $\mathrm{NaCl}$ for an additional 3 weeks.

has already been established (figure 2A). When started 3 weeks after the onset of bleomycin challenge, doses of $0.3 \mathrm{mg} / \mathrm{kg}$ oral daily pomalidomide completely prevented further dermal thickening despite ongoing challenge with bleomycin (figure 2B). At doses of $30 \mathrm{mg} / \mathrm{kg}$ oral daily, pomalidomide did prevent progression and induced regression of bleomycin-induced fibrosis and decreased the dermal thickness below pretreatment levels despite ongoing injections with bleomycin $(p=0.03$ compared with sham-treated mice challenged with bleomycin for 3 weeks followed by injections of $\mathrm{NaCl}$ for an additional 3 weeks) (figure 2B). Myofibroblast differentiation and accumulation of collagen were dose-dependently reduced below pretreatment levels (figure 2C,D).

\section{Treatment with pomalidomide decreases the levels of TGF $\beta$-target genes in fibrotic skin}

A common characteristic of both models is the activation of transforming growth factor (TGF) $\beta$ either due to increased release of TGF $\beta$ as in bleomycin-induced dermal fibrosis or due to increased activation of latent TGF $\beta$ as in Tsk-1 mice. ${ }^{8}$ Treatment with pomalidomide significantly decreased the mRNA levels of the classical TGF $\beta$-target genes CTGF and PAI- 1 as well as colla1 in Tsk-1 mice as well as in bleomycin-induced dermal fibrosis (figure $3 \mathrm{~A}-\mathrm{F}$ ), indicating that inhibition of TGF $\beta$ signalling may contribute to the antifibrotic effects of pomalidomide.

\section{DISCUSSION}

The present study demonstrates that the IMiD pomalidomide is effective in different preclinical models of dermal fibrosis. Of note, pomalidomide did prevent fibrosis and induced regression of pre-established experimental dermal fibrosis in therapeutic settings. The mechanism of action of pomalidomide in these diseases is thought to occur through a combination of immunomodulatory, antiproliferative and other mechanisms. ${ }^{3}$ Recently, the molecular binding target of pomalidomide has been identified as cereblon, a component of an E3 ubiquitin ligase complex controlling protein ubiquitination. ${ }^{13}$ As both the immunomodulatory and tumour antiproliferative effects of pomalidomide appear to be dependent upon cereblon, this target may be responsible for other pharmacological effects of pomalidomide as well. In the dermal fibrosis studies described herein, the mechanism of action of pomalidomide may be through a combination of immunomodulatory and antifibrotic effects. For example, in the pre-established bleomycin-induced fibrosis model, treatment with pomalidomide resulted in an increase in serum levels of the Th1 cytokines interferon $\gamma$ and tumour necrosis factor $\alpha$, and a decrease in the Th2 cytokine 
A

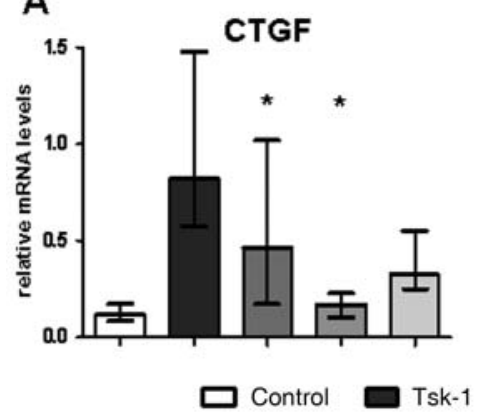

B

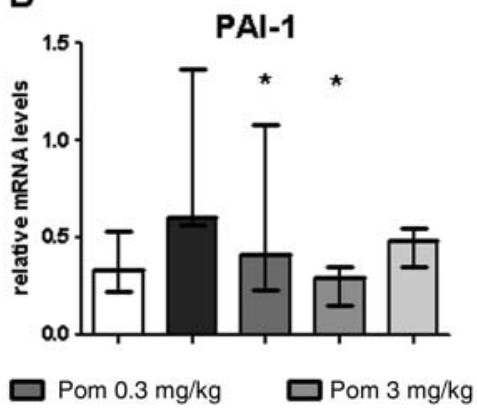

C

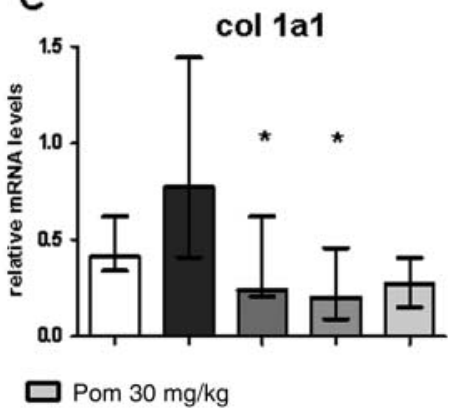

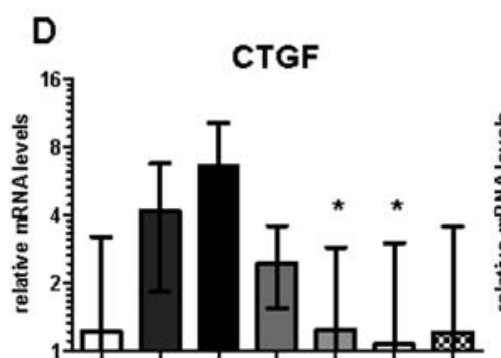

\section{E}

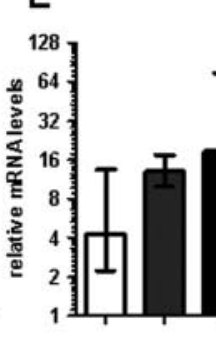

PAl-1

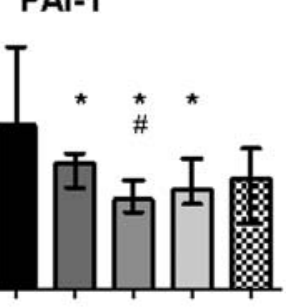

bleo 3 weeks; $\mathrm{NaCl} 3$ weeks

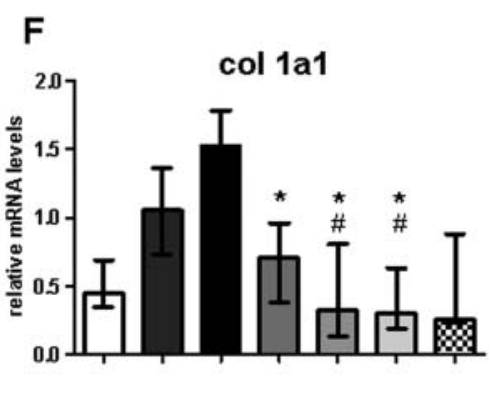

$\square$ Pom $3 \mathrm{mg} / \mathrm{kg}$ last 3 weeks

$\infty$ Imatimib $50 \mathrm{mg} / \mathrm{kg}$ last 3 weeks

Figure 3 Pomalidomide (Pom) decreases the mRNA levels of TGF $\beta$ responsive genes in experimental fibrosis. Treatment with Pom decreases the mRNA levels of CTGF (A), PAl-1 (B) and col 1a1 (C) in Tsk-1 mice as well as in the model of bleomycin (bleo)-induced established fibrosis (D-F). $\mathrm{N}=6-8$ for all groups. ${ }^{*}$ Indicates statistically significant changes compared with sham-treated pa mice or sham-treated mice challenged with bleo for 6 weeks, respectively. ${ }^{\#}$ Indicates statistically significant changes compared with sham-treated mice challenged with bleo for 3 weeks followed by injections of $\mathrm{NaCl}$ for an additional 3 weeks.

IL-5. Of note, the stimulatory effects of pomalidomide on tumour necrosis factor $\alpha$ were not associated with increased, but with decreased TGFß signalling.

Additional evidence for the antifibrotic effects of IMiDs is provided by studies with the ancestor compound thalidomide, which has less potent immunomodulatory effects and a less favourable safety profile compared with pomalidomide. ${ }^{2}{ }^{3}$ Thalidomide demonstrated efficacy in experimental pulmonary and liver fibrosis and also ameliorated postoperative bowl adhesions as well as remodelling after myocardial infarction in preclinical models. ${ }^{14-18}$ Beneficial effects of thalidomide were also reported from a shortterm open-label trial of thalidomide in scleroderma patients. ${ }^{19}$ Although conclusions on efficacy are not possible from this trial due to the limited number of patients, the lack of a control group, the heterogeneity of the participants and in particular the lack of direct clinical or molecular parameters of fibrosis, this study highlights that the use of IMiDs in scleroderma may not be limited by side-effects such as the inhibitory effects on vascular endothelial growth factor (VEGF) signalling. Considering the potent antifibrotic effects of the IMiDs thalidomide and pomalidomide and encouraging effects of pomalidomide in myelofibrosis and chronic graft-versus-host-disease (cGvHD), ${ }^{2} 320$ pomalidomide may be an interesting candidate for clinical studies in patients with fibrotic diseases. Indeed, clinical trials with pomalidomide have recently been initiated in cGvHD (www.clinicaltrials.gov). In addition, an international, multicentre, controlled phase II clinical trial to evaluate the safety and the tolerability of pomalidomide in SSc has most recently been launched (clinicaltrials.gov ID: NCT01559129).

In summary, pomalidomide exerts antifibrotic effects in preclinical models of dermal fibrosis and prevented fibrosis and induced regression of pre-established fibrosis. Our findings have direct translational implications and served as drivers of clinical trials with pomalidomide in SSc.

Acknowledgements We thank Anna-Maria Herrmann, Madeleine Demleitner, Verena Wäsch and Stefan Fritz for excellent technical assistance.

Contributors SS, PS, JHWD: design of the study; SW, PZ, MT, KP-Z, AD, CD, CB, SS, DC, PS: acquisition of data; SW, PZ, KP-Z, SS, DC, PS, OD, GS, JHWD: interpretation of data; SW, PS, JHWD: manuscript preparation.

Funding Grants DI 1537/1-1, DI 1537/2-1, DI 1537/4-1, DI 1537/5-1, AK 144/1-1 and SCHE 1583/7-1 of the Deutsche Forschungsgesellschaft, grants A20 and A40 of the IZKF in Erlangen, the ELAN-Program of the University of Erlangen-Nuremberg and the Career Support Award of Medicine of the Ernst Jung Foundation and by support of CMH Research Projects No 00000023728.

Competing interests The study was supported by Celgene Corporation. Sai L Shankar, Dorota Cedzik and Peter H Schafer are employees of Celgene Corporation.

Provenance and peer review Not commissioned; externally peer reviewed.

\section{REFERENCES}

1. Gabrielli A, Avvedimento EV, Krieg T. Scleroderma. N Engl J Med 2009;360:1989-2003.

2. Knight R. IMiDs: a novel class of immunomodulators. Semin Oncol 2005;32: S24-30.

3. Galustian C, Dalgleish AC. Pomalidomide. Drugs of the Future 2011;6:741-50.

4. Corral LG, Haslett PA, Muller GW, et al. Differential cytokine modulation and T cell activation by two distinct classes of thalidomide analogues that are potent inhibitors of TNF-alpha. J Immunol 1999;163:380-6.

5. Davies FE, Raje N, Hideshima T, et al. Thalidomide and immunomodulatory derivatives augment natural killer cell cytotoxicity in multiple myeloma. Blood 2001;98:210-16.

6. Keifer JA, Guttridge DC, Ashburner BP, et al. Inhibition of NF-kappa B activity by thalidomide through suppression of IkappaB kinase activity. J Biol Chem 2001;276:22382-7. 
7. Xu W, Celeridad M, Sankar S, et al. CC-4047 promotes Th1 cell differentiation and reprograms polarized human Th2 cells by enhancing transcription factor T-bet. Clin Immunol 2008;128:392-9.

8. Beyer C, Schett G, Distler 0, et al. Animal models of systemic sclerosis: prospects and limitations. Arthritis Rheum 2010;62:2831-44.

9. Dees C, Akhmetshina A, Zerr P, et al. Platelet-derived serotonin links vascular disease and tissue fibrosis. J Exp Med 2011;208:961-72.

10. Akhmetshina A, Venalis $P$, Dees $C$, et al. Treatment with imatinib prevents fibrosis in different preclinical models of systemic sclerosis and induces regression of established fibrosis. Arthritis Rheum 2009;60:219-24.

11. Avouac J, Furnrohr BG, Tomcik M, et al. Inactivation of the transcription factor STAT-4 prevents inflammation-driven fibrosis in animal models of systemic sclerosis. Arthritis Rheum 2012;63:800-9.

12. Avouac $\mathbf{J}$, Palumbo K, Tomcik M, et al. Inhibition of AP-1 signaling abrogates TGF-ss mediated activation of fibroblasts and prevents experimental fibrosis. Arthritis Rheum 2011;64:1642-52.

13. Zhu YX, Braggio E, Shi CX, et al. Cereblon expression is required for the antimyeloma activity of lenalidomide and pomalidomide. Blood 2011;118:4771-9.
14. Choe JY, Jung HJ, Park KY, et al. Anti-fibrotic effect of thalidomide through inhibiting TGF-beta-induced ERK1/2 pathways in bleomycin-induced lung fibrosis in mice. Inflamm Res 2010:59:177-88.

15. Mall JW, Schwenk W, Philipp AW, et al. Thalidomide given intraperitoneally reduces the number of postoperative adhesions after large bowel resection in rabbits. Eur J Surg 2002;168:641-5.

16. Muriel P, Fernandez-Martinez E, Perez-Alvarez V, et al. Thalidomide ameliorates carbon tetrachloride induced cirrhosis in the rat. Eur J Gastroenterol Hepatol 2003;15:951-7.

17. Yndestad A, Vinge LE, Bjornerheim $R$, et al. Thalidomide attenuates the development of fibrosis during post-infarction myocardial remodelling in rats. Eur J Heart Fail 2006;8:790-6.

18. Zhao L, Xiao K, Wang H, et al. Thalidomide has a therapeutic effect on interstitial lung fibrosis: evidence from in vitro and in vivo studies. Clin Exp Immunol 2009;157:310-15.

19. Oliver SJ, Moreira A, Kaplan G. Immune stimulation in scleroderma patients treated with thalidomide. Clin Immunol 2000;97:109-20.

20. Pusic I, DiPersio JF, Goran SL. Phase 2 study of pomalidomide in advanced corticosteroid-resistant chronic graft-versus-host disease (cGVHD). American Society of Hematology 51st Annual Meeting and Exposition 2009; December 7. 


\section{ARD Pomalidomide is effective for prevention and treatment of experimental skin fibrosis}

Simon Weingärtner, Pawel Zerr, Michal Tomcik, Katrin Palumbo-Zerr, Alfiya Distler, Clara Dees, Christian Beyer, Sai L Shankar, Dorota Cedzik, Peter H Schafer, Oliver Distler, Georg Schett and Jörg H W Distler

Ann Rheum Dis 2012 71: 1895-1899 originally published online August 17,2012

doi: 10.1136/annrheumdis-2012-201784

Updated information and services can be found at:

http://ard.bmj.com/content/71/11/1895

These include:

Supplementary Material

References

Email alerting service
Supplementary material can be found at:

http://ard.bmj.com/content/suppl/2012/08/16/annrheumdis-2012-2017 84.DC1.html

This article cites 19 articles, 5 of which you can access for free at: http://ard.bmj.com/content/71/11/1895\#BIBL

Receive free email alerts when new articles cite this article. Sign up in the box at the top right corner of the online article.

Topic Articles on similar topics can be found in the following collections

Collections

\section{Notes}

To request permissions go to:

http://group.bmj.com/group/rights-licensing/permissions

To order reprints go to:

http://journals.bmj.com/cgi/reprintform

To subscribe to BMJ go to:

http://group.bmj.com/subscribe/ 